\title{
Long Term Learning Of AgILe Teams
}

\author{
M. Rizwan Jameel Qureshi and Zahra Abass \\ Faculty of Computing and Information Technology, \\ King Abdulaziz University, Jeddah, Saudi Arabia
}

\begin{abstract}
The agile software development methods have demonstrated success in increasing responsiveness to the needs of customers. So the most of software industry preferred the agile methods. These methods have increased demand by the companies, but the agile methods at larger scale are more challenging and problematic compared to the smaller projects. For that, to become the norm for a large and complex project a new adaptive agile framework is proposed. Also, the proposed framework will help to achieve long-term organizational and learning of agile teams and help to increase the employee motivation and satisfaction by improving their skill. Thus the organizations will have success in sustaining their team and increasing its productivity.
\end{abstract}

\section{KEYWORDS}

Agile methodologies, continuous learning, knowledge sharing, agile team, long-term learning

\section{INTRODUCTION}

Organizations are confronting always developing conditions and changing requirements of clients. This change requires new software development techniques that empower adaptability and versatility keeping in mind the end goal to agree to the frequently changing requirements of clients. Customary, arrangement based systems are no longer most appropriate in the greater part of these evolving situation. This requirement for adaptability and flexibility prompted the developing prominence of agile methodologies [1].

Agile Software Development is a gathering of methods of software development that advance self-organizing team, adaptive planning fast and continuous delivery, and client focus. Methodologies of agile support constant change and fast, sufficient reaction to change . Agile methodologies are particularly intended to encourage collaboration, coordination and communication in an environment of dynamic team. This incorporates practices, for example, shared team rooms to empower visit casual communication, useful work environment conditions to permit pervasive data scattering, and quick cycles of feedback through which a product item is advanced incrementally in close association with a client agent. Rather than adhering to traditionally long periods of upfront requirements gathering and design before software production, agile teams elicit feedback early on in the process, and deal with the complexities of software development by practicing rapid iterative development from project inception [2].

These changing requirements don't just concern small projects and organizations, but larger projects and organizations too. This appears to be logical since similar issues tended to in Agile methodologies are faced by large organization [1].

DOI: $10.5121 /$ ijsea.2017.8601 
Nonetheless, Agile methodologies were initially intended for, and considered fruitful, for small project and teams, which makes it not clear that large, complex organizations and projects may effectively apply the method of Agile.

So, Agile software development methodologies are successes and applied for the small and teams and projects. One of the most significant challenges is scaling up these methods and also the large organizations facing the same challenges that agile methodologies have addressed.

Three of the most vital difficulties in scaling up Agile methods are changing leadership requirements, communication barriers and unaligned, ineffective teams.

For that, the agile methodology based on a team performance. Thus setting up team is most important in innovation [3].

So, this research is written to address this question: "how to achieve a long-term learning and organizational goals of agile teams to minimize the challenges of scaling agile in medium and large organizations?". To address these software industry problems, a new adaptive, agile framework is proposed that is appropriate for medium and large software organizations. Also, this agile framework will help in improving organizational productivity, increasing the satisfaction of teams by improving their skills and personal communication when sharing the knowledge and learning activities and achieve a long term organizational goals.

\section{RELATED WORK}

Realize and identify success factors that influence on the success of agile projects and teams is important.

\subsection{Communication And Collaboration}

Agile methods focus on face to face communication that is important factor to the collaboration of an agile team [6]. Therefore, a framework is proposed which aimed to study the collaboration driven by the requirement between the agile team [6]. For formalized the framework, the data is collected by semi-structural, interviews, questionnaires and on-site observation. At two software development organizations a multiple case studies are conducted to demonstrate the framework particularly. The finding of study the proposed framework is aided in defining the collaboration trends, core members, clustering tendency, small worldliness and centralization behavior of the networks, communication and awareness reciprocity of the teams and iteration performance of the agile teams. In addition, the framework is helpful for practitioners of industrial to measure the performance improvement by learning about their team collaboration.

In another effort to improve the productivity of large software companies, agile methods are employed to be successful in addressing the demands of customers more rapidly. However, a multiple agile teams suffer from unnecessary inter team interactions that may lead to paralysis. So a framework is guided to identify factors that causing effect of interactions between teams and generate the speed waste. Also, the study has provided some of the recommendations to manage such factors in order to complement practices of agile, as a result a large software companies can be adapted by these recommendations. The factors and recommendations in this research analyzed in term of interaction speed and other impacts not taken into consideration [7]. 
Beside to interactions that based on speed as a success factor for agile team, it is important to identify which other success factors that influence on the success of agile projects and teams. Therefore, a conceptual model is proposed based on literature and interviews held with best practices [5]. The proposed model is tested quantitatively on a set of 40 projects from 19 Dutch organizations, comprising a total of 141 project members, Scrum Masters and product owners. Subsequently, the result of study has identified the important success factors in the agile project success that are degree of adoption of agile practices, value congruence and transformational leadership. Also the study is found that agile project success cannot influence by the project size, thus agile methods seem to be successfully applied in larger projects, as long as there is transformational leadership, agility and high value congruence.

Furthermore, Melo et al. [8] conducted two industrial case studies investigate the factors that influence on the productivity of an agile team. The factors are external dependencies, team composition and allocation and staff turnover. The authors extended and refined this work to provide a better understanding of some factors [9] that impact the productivity of an agile team. So, this extension contained a one larger company is added to the other companies in the previous paper and two source data for the three companies. Accordingly, a conceptual framework for the productivity of an agile team is proposed by analyzing thematically based the data sources that were extracted from multiple studies. Consequently the result of this study is an agile team management founded as the important influence factors on productivity of an agile team. The member turnover and team design (structure and work allocation) are the main factor productivity of the intra team level. Also, avoiding delays in delivering promised software to dependent team and the effectively coordinated to the team by dependencies and proper interfaces are the main factor productivity of the inter team level. The result concluded that the team must be aware of the magnitude and influence of turnover, which are negative for the productivity of an agile team. In addition an important factor that impact team productivity is team design choice. So the processes of inter team coordination should be adjusted by considering the pace between team and priorities to enable productive work.

For ensuring the success of the project, an agile project manager playing an important role because he is accountable for the success or failure of the project. So, he must take into consideration how to deal with any problem by adopting agile methodologies in the project [4]. Therefore, seven behaviors are identified for the manger when adopt the agile in managing the software development process. They are openness, leadership, ethics, communication, creative and innovative, results orientation and strategic. Also, taxonomy of behavior for the agile project manager is conducted that helped the practitioners to consider them that promoted their project success. Accordingly, the profitability and productivity of software development project will be increased. But the study has not implemented and need more research to be validated.

\subsection{SELF-ORGANIZING TEAM}

The heart of agile software development is self-organizing teams that are the best design, requirements and architecture and have been identified as one of the significant success factors for agile projects [10]. So a Ground Theory study is conducted for 23 different organizations that involving of 58 agile practitioners [10]. The result of the study is self-organizing teams influenced by the senior management. Senior management is important to support self-organizing in term of critical factors such as maintaining and creating an informal and open organizational culture, providing financial sponsorship, negotiating "Agilefriendly" contracts and managing human resources in a way that supports self-organization. But the senior management without 
managing to these factors efficiently leads to challenges for self-organizing teams. In addition, the authors in this research have improved his study to identify the implicit, informal, spontaneous and transient roles. The roles are a Coordinator, Mentor, Translator, Promoter, Champion and Terminator that focused toward providing encouraging continued adherence to agile methods and providing initial guidance, coordinating customer collaboration and effectively managing customer expectations, sustaining and securing senior management support and identifying and removing team members threatening the self-organizing ability of the team [11].

In agile projects, self-organizing is perceived as one of the most significant success factors [16]. Dingsoyr, Moe et al [17] have done studies on the of self-organizing obstructions in an agile team. They discovered issues to associate with both organizational context and team particular factors. On organizational context the structure for sorting out work culture and, control assumed most important roles while on team level the difficulties were identified with responsibility, inability to learn and to decision making and basic leadership parts and examples. While considering the difficulties of team level, missing team structure and absence of clear and shared objective among team members to encourage cross-usefulness debilitated self-organizing abilities. Promote on the team need to practice that they really have independence to decide. The decision-making absence has been identified with learning about the team since having the likelihood to likewise commit falls and mistakes as a team over the long haul in reality encourages the learning of the team. At long last, winning old initiative mindset that inclines toward single specialists and individual objectives made critical issues that occur in a team about self-organizing, so it forestalled interaction, shared decision making and communication to happen in the team. While the organizational context is considered, there are greatest obstructions comprises for example, operational structures that prevented teams from shaping and remaining stable, an organization that did not add to delivering the project lastly such culture or progression that underscored singular achievements over team accomplishments.

Having trust among team members, shared concentration and capacity to revamp have been recognized as critical variables for self-organizing in agile team and a huge issue identified with this in both organizational level and team identifies with trust and communication. On the off chance that team members don't convey and share information, they have propensity to work in separation from each other, which diminishes repetition and anticipates the learning of team learning. Trust has been seen as basic consider ensuring team's self-organizing capacity, since it diminishes chain of importance and administration from organizational context and enhances the sharing of information amongst the members of [18]. of project lacking organizational trust is a circumstance where a team needs to deliver advanced outcomes to the stakeholders of the project, preventing them from focusing on the actual objectives that they have. This is requiring pointless exertion from team to impression administration and the issue identifies with organizational trust towards team to deliver. Promote on absence of trust inside team members can bring about impression administration even inside a team, creating team members to conceal their blunders and averting real performance improving and feedback. Sign of lacking trust can likewise develop as "decision hijacking" where decisions are not made altogether, but rather by a single team member who hopes to have the required information or specialist to decide [17]. This sort of conduct, further decreases the accomplished trust inside team and furthermore decreases motivation to take initiative with regards to the team.

In agile projects, It appears the culture of shared responsibility is winning, there is frequently disjointedness with respect to responsibility anticipating move making in the team. This can come from amorphous role definitions additionally thinking paradox of group. So as to defeat these 
International Journal of Software Engineering \& Applications (IJSEA), Vol.8, No.6, November 2017

difficulties, the team should to guarantee there is a procedure to both implement and make decisions. Researchers have expressed [19] that in actuality the operationalization in decision making is more vital that the way toward achieving the decision, showing that numerous great decision come up short in view of lacking usage. To enhance the decision procedure and conquer the difficulties of going for accord, teams ought to practice to concede to the most reasonable individual to claim the issue, allot activity focuses for every decision and furthermore look for sufficiently early help likewise from project stakeholders. This however requires teams are capable to identify the issue and its significance is perceived [19]. It is likewise imperative that there is culture to notice challenges when they show up, and regular meetings, for example, every daily meetings are utilized to spot issues requiring consideration [18].

\subsection{CROSS-FunCTIONAL TEAM}

For every stage of developing software, agile team must be cognizant of all expertise necessary for them. So a cross-functional team is intended for agile teams. Therefore, the other expertise which is located outside the team is necessary for an agile team, for instance, Database Administrators (DBAs), User Experience Designers and Software Architects [12]. External specialist expertise is valuable to support agile teams with specialized skills. The author has applied a grounded theory for 47 external specialists and agile practitioners to explore the relationship between them.

Consequently, the result of study is five factors are needed when coordinating external experts for external specialists and agile practitioners which are available, agile mindset, stability, knowledge retention and effective communication. Also the paper presented a guideline for coordinating external expertise in the team by strategies for each factor. The study is important to help agile teams to utilize external resources effectively.

Knowledge sharing is important to enhance cross-functional team performance [15]. Accordingly, Kavitha an Irfan developed a framework to share and capture the Knowledge among agile team. The proposed framework facilitates the learning process by maintaining and capturing the important knowledge. Thus, the success of the software development depends on the developer expertise and knowledge [12]. In addition shared tasks and knowledge is needed for team learning.

In [14] presented agile team learning model (ATLM) that based on the fast tasking mining. So this model is contributed to develop a teamwork ability of team member and also proposed a fast tasking mining method which is important to shorten the learning period and develop the knowledge acquired. The model is applied for small project not take into consideration the large companies.

As well, for long lasting progress of the organization, the continues of long term learning is important. For that Ourashi at el [24]. proposed agile process model that provided an excellent opportunity to help in satisfaction, motivation, presentation, coordination and enhancement of agile team by technical skills. In addition, this process of long term learning are helping to make organizations to save their success and lead the team members and organization to successfully market lead.

A research thinking of agile team learning depend on rough set mode (R-S mode) and scenario analysis is proposed in []. It is significant to the team learning in unconventional emergency 
International Journal of Software Engineering \& Applications (IJSEA), Vol.8, No.6, November 2017

decision making to find an efficient analysis method. Besides, this work shows the frame of agile team learning and analyzed agile team learning characteristics in decision making about unconventional emergency.

Moreover, reflection is an important part of learning cycle that was encouraged by many of agile proponents. Thus, some techniques are discussed in [23] that help to overcome the initial reluctance that is important to taking reflection to a deeper level and keeping reflection meetings interesting.

\section{Problem Definition}

Agile methods are generally utilized for organizations that have a small project, and most accessible contextual analyses analyzing their usage are centered around small organizations using a single-team structure. Agile methods are practically quiet about large software organizations with different teams and their communications. In agile methods, there are no rules on the best way to defend long haul hierarchical objectives inside a similar umbrella. Various analysts endeavored to scale agile methods for medium and huge activities, however, numerous imperative parts of scaling are lost, for example, learning, aggressive edge, center, and reusable contemporary information. At times, by implanting organized methodologies and acquiring basic leadership values in software organizations of the agile method to pick up/support the part of the market pioneer.

\section{The Proposed Adaptive, Agile Model}

This section will present the proposed model that will be help in solving some of the challenges that was aforementioned also will present how collect the data and analyzed it. Rotational learning for leadership is a heart to bring about the organization support ability and advance by change of the information stream, change of specialized aptitudes, change of representatives leadership qualities, inspiration and fulfillment of them and coming about lower wear out, disassembling organized organization and basic leadership.

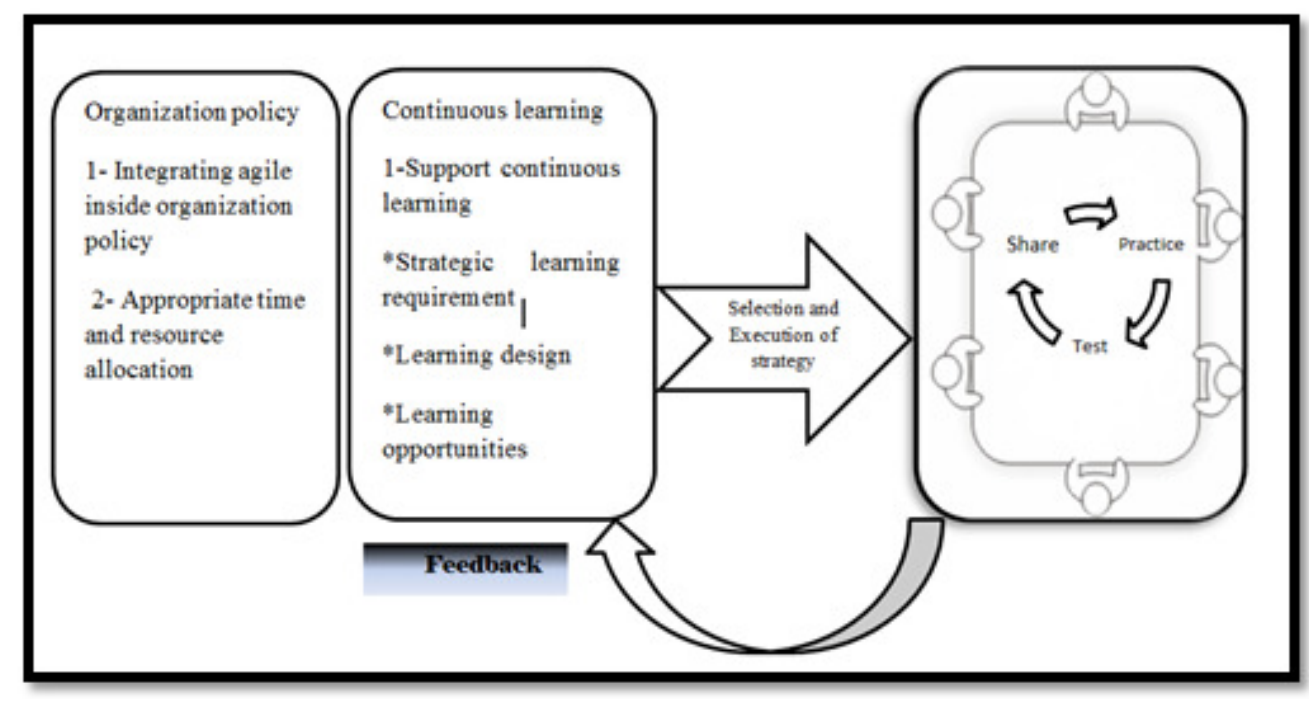

Figure 1. The proposed adaptive model 
International Journal of Software Engineering \& Applications (IJSEA), Vol.8, No.6, November 2017

\subsection{Organization Policy}

\section{1-Integrating agile inside the organization policy}

For the sake of growing organization productivity, increasing the satisfaction of employees through stimulating their communication skills, sharing of knowledge, learning activities, and accomplish a durable organizational goals, a stand-in team should be promptly created, trained, proficient and sustained. These thoughtful defies, together with the effect of culture-changing know-hows, the necessity to rethink, redesign and retool (3Rs) the shared learning atmosphere should be initiated. Learning might perhaps not much be restricted to tutorial room, otherwise eeducation and further learning approaches. Incorporation, swiftness, scope and concurrent communication should be created in the learning setting. To build an agile sustainable learning, preparation, culture-base and knowledge-distribution, the company polices and plans should combine with design and content. However, the approach we think regarding the learning process should change. Many organizations can truly realize substantial benefits after they integrate various learning approaches.

So, as agile is installed in strategic documents which will be utilized for the long-term basis, along these lines, coordinated will turn out to be an integral part of the organization's policy. It will be utilized as a part of long hurried to get profits from it. The main thing which should be possible is the rule and this rule as the announcements will assist to make decisions for decision makers as per the rules of agile. Accordingly of it coordinated will be utilized as a part of the long keep running on a long term basis. The express specifying of agile in the key records set line of activity clear for an organization. It will draw limits for the organization how to move, how to control and how to create and keep up programming in the evolving condition. It will likewise assist an organization to take care and think about issues in a coordinated way. Teams of agile are cross-functional teams. They don't have any unequivocal learning part. It is because of the brief time nature of the agile. In any case, when the organization is huge and having the numerous teams and project, what should do with the project support in light of the fact that no or little documentation will be accessible. In a small agile team, it is not the issue for the same people who have built up the project may at present be accessible to adjust or change the product as indicated by the client needs. Be that as it may, in the multi-team and multi-project, this will end up being a scam as individuals will continue moving to and from the organization. This will be the first phase and will be applied one time.

\section{2-Appropriate time and resource allocation:}

Time allocation, here, is the whole period of time available for learning, for instance, the length of the training period. The Gantt chart can be used as an effective technique to allocate precise blocks of time to the various tasks in the adaptive agile framework. On the other hand, resource allocation is the assignment of the company available resources to achieve long-term learning goals.

\subsection{The Continuous Learning}

\section{1-Support continuous learning:}

After that, the second phase will be begin with continuous implementation with a list of requirements for strategic organizational learning that will be produced and will continue 
International Journal of Software Engineering \& Applications (IJSEA), Vol.8, No.6, November 2017

changing due to the new and developing innovations. There are a few rules for the choice of learning requirements yet they are not thorough. Advertising arrangement generally created on the premise of many components, of which some significant variables are organization skills, strategic planning, competitive factors and so on. Actually, it is a perfect archive that can lead us legitimate discovery of requirements for learning. This will likewise bring coordination between various divisions of the organization to anticipate the risks of absence of coordination. Consider the circumstance in which showcasing team is advertising to locate the new business with apparatuses of Oracle while another division might go for Java advancements. In the event that promoting group prevailing to get great business yet the advancement group has nothing to do it. Agile technique excellence lies in the broad individual association. Agile is individuals driven and demoralize the documentation when an issue can be illuminated with up close and personal collaboration. Scrum is an agile strategy and it has diverse sorts of broad meeting.

Amid these broad organization learning desires can be judged. For instance, if a man is all the more ready to perform errands identified with the databases and less keen on interface advancement then it can be judged that he is more suited to prophet database programming than the front end programming.

Learning opportunities implies any remarkable undertaking that can give new figuring out how to the general population inside or outside of the organization (preparing and course). The fundamental concentration of our strategy will be to discover such open doors inside the organization. A graph can be made and each group which makes them learn opportunity will post it on the diagram. The proposed design of the diagram comprises of a concise depiction of learning opportunities and the normal date of the beginning alongside its length and place.

We have a list of strategic learning requirements. Secondly, a list of learning desires is also available. A list of the learning opportunities is also there.

Those learning desires which coordinate with future work requirements will come about into restricted bringing down on the learning list. On this list a further intersection is connected with learning opportunities found in the organization. This will come about into a definite list giving the information which team members must be prepared what (long term learning requirements) and where (learning opportunities within the organization).

Keeping in mind the end goal to, don't hurt agile spirit and evading any aggravation in the organization, it is suggested that exclusive few the representatives learners might be in this procedure at any given time. This learning procedure will come about into followings.

As this learners won't just take on the particular innovation additionally the space information on the project and distinctive culture and working style. To be sure he will likewise impart his own particular encounters to this team. In the wake of coming back to another team, he will likewise share his discovering that will keep up the stream of learning. At the point when this movement will be performed on consistent premise, it will bring about the steady stream of the information in the entire organization.

Impacts are an endeavor to quantify whether the proposed model has any significant effect on the organization to get and maintain the market authority through learning for administration. At the point when a team member will move starting with one team then onto the next team as a mentor, he will communicate with other team members. The coach will comprehend the way of life and 
International Journal of Software Engineering \& Applications (IJSEA), Vol.8, No.6, November 2017

style of new team and he will impart his insight and experience to the new team to keep up the stream of information. Thusly, various teams will know about them. Correspondingly, when a coach will move to some other team then he will be in a position to associate with individuals from another team in a vastly improved manner. Consequently, the communication between the teams will be improved because of the learning for authority.

Additionally, agile teams must occupy with continuous learning through community-learning, self-learning or peer-learning, to increase particular information that is by all accounts imperative or required for software advancement exercises. Learning additionally occurred through interaction between team members or individuals from different teams in the organization. The administrators ought to be comprehended that colleagues required distinctive knowledge of various circumstances to address the issues of the product advancement activities.Therefore, supervisors obviously supported self-learning and gave a team member self-learning opportunities.

\section{2-Use latest learning and knowledge-sharing activities:}

The entire development and learning activities should be attentive and purposefully integrated through the principal learning department's predominant learning policy. A learning policy which unites the entire facets of shared learning not merely will swiftly run away from the teaching space, however, similarly will offer teams with more self-control over the opportunities of learning that incorporating, completing and enhancing proper learning and training environment with sustainable sharing of knowledge among the team. Applying a multi-dimensional learning methodology, courses are incorporated into the culture-based learning atmosphere and are not isolated procedures. Concentrating on performance and the whole requirements of the team, learning must be applied to deliver fundamental skills and abilities, meta-knowledge, know-how technologies and the competence to access knowledge twenty-four-hour-a-day.

\section{3-Implement a thoughtful long-learning Strategy:}

A significant driver for long-learning strategy is the desire of the company to develop and retarget prevailing learning content which need to be restructured, rationalized and transformed into a company-based sophisticated courses. A firsthand agile learning setting should take into account immediate access to subject-matter professionals, an acquaintance at the time of necessity and onthe-job right of entry to the whole pertinent learning tools and practices. As part of a knowledgesharing approach, organizations can emphasis on the learning-on-appeal requirements of the teams.

\subsection{MANAGING KNOWLEDGE}

After the previous phase teams must to understand how to managing knowledge where the continuous learning is a significant phase to help in executing of this part. So, some finding that emerged when applied the continous learning. 


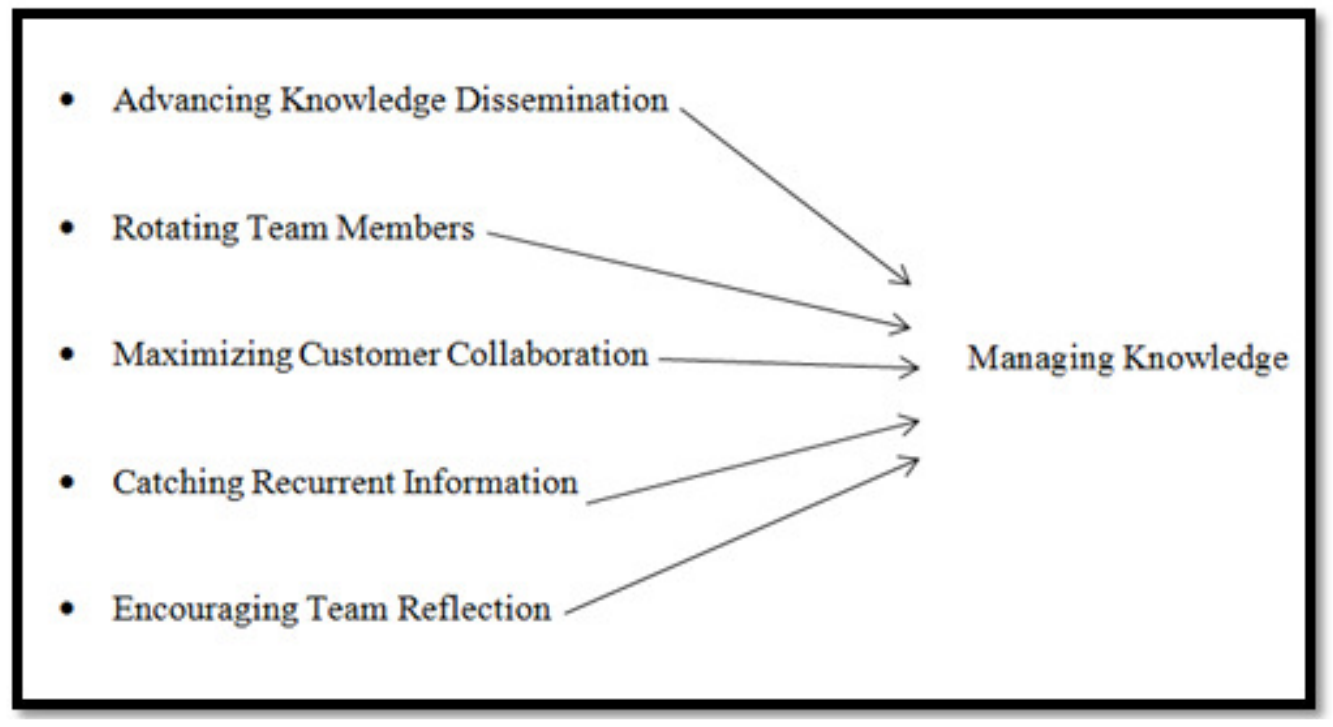

Figure 2. Managing Knowledge

\section{1-Advancing Knowledge Dissemination}

Agile team must take part in the everyday meeting that brought team members together to synchronize the work of all team individuals, distinguish conceivable obstructions to the project advance, and timetable future meeting. So the daily meeting is more huge to fill in activity of team building that will make the individuals feel some portion of the group, especially when the meeting will include all individuals from locations of a distributed team. Team members must comprehend that an everyday meeting may satisfy its motivation just when important information that advanced comprehension and learning is shared with the team.

\section{2-Rotating Team Members}

Information sharing between appropriate team members occurred amid daily meeting through technology mediated communication such sound or video conferencing.Face-to-face discussion was, be that as it may, considered the most proficient and compelling technique for passing on data. At the point when and as required, cross-area visits gave chances to up closer and personal communication between appropriate individuals to increase better comprehension of the basic circumstances in the product advancement ventures Cross-area visits more often than not occurred for a brief timeframe. Its primary reason for existing was for gaining information required for programming advancement yet the wellspring of learning was accessible just in another area. Rather, rotating team members across over various areas to advanced, dependable learning exchange over all areas.

\section{3-Maximizing Customer Collaboration}

Agile team is a cross-functional team that is staffed with skilled individuals, including clients or client delegates, performing obliged parts to manufacture a product and add to the achievement of a project. At the point when clients chipping away at the site with the advancement groups, learning, sharing will be upgraded through frequent communication, face-to-face interaction and close collaboration between individuals from the improvement team and the clients. 
International Journal of Software Engineering \& Applications (IJSEA), Vol.8, No.6, November 2017

At the underlying phase of a project partners must sort out a beginning workshop which is an intuitive arrangement of organized workshops that give chances to partners to talk about the project objectives and vision, business benefits, costs, plan, achievement measures, innovation presumptions, and principles of engagement. Amid the beginning workshop, teams ought to discuss about collaboration, engagement and communication plans amongst improvement teams and clients.

\section{4-Catching Recurrent Information}

Team members frequently requested information, either project particular information or general, specialized information, from topic specialists. Incidentally, topic specialists request a similar information over and over through the span of a project, frequently by various people in the team instead of a similar person. Consequently, distributed teams must make an interpretation of tacit knowledge into explicit knowledge in the form audio, video or form of written for storage in repositories of knowledge. These information relics spoke to the repetitive information required for a project team, and reliably recovered and utilized when and as required.

Another member who joined to the teams will be furnished with adequate information required for him to begin with, his occupation through the information caught and put away in the knowledge repositories. This information is likewise helpful for existing individuals who requesting a similar information at a different period of time.

\section{5-Encouraging Team Reflection}

Agile teams ought to be reflected at regular intervals, keeping in mind the end goal to enhance development processes, forms and change the conduct of team member. Reflection likewise encouraged communication and openness between team members, and it is especially vital for distributed members to understand collaboration restriction and communication limitation. At the point when distributed teams occupied with incessant dialogs, team members can increase sound information on the Agile practices, innovations and general programming improvement forms utilized in the projects. Through regular dialogs clients and subject matter experts, team members are furnished with chances to refine, re-organize, and re-create prerequisites and arrangement.

This study was separated into the following six objectives to assess the proposed model.

1. Agile as integral part of the organization's policy

2. Allocates appropriate time and resources as the demand of the organization and its teams and measure their effect on the learning.

3. Continuous learning is essential category for the organization and employees and measuring its effected.

4. Measuring the effect of learning from leadership to the other teams in the organization.

5. Measuring the effect of the steps to manage the knowledge and its significant on Spread the learning.

6. Assessing the implementing of the proposed model to achieve the long term learning of whole teams in a medium and large organization.

\section{Collect And Analyze The Data}

Descriptive research can be clarified as a statement of affairs as they are at present with the researcher having no power over variable. In addition, " Descriptive research might be portrayed 
as essentially the endeavor to decide, depict or recognize what is, while analytical research endeavors to set up why it is that way or how it came to be"[22].

Descriptive research is "aimed at throwing light on flow issues or issues through a procedure of data collection that empowers them to depict the circumstance more totally than was conceivable without utilizing this method"[22]. This type of research describes what exists and may reveal new actualities and significance. The motivation behind descriptive research is to describe, observe, archive aspects of a situation as it normally happens (Polit and Hungler 1999). This includes the collection of data that will give a record or depiction of people, gatherings or circumstances. The instruments we use to get data in in descriptive studies include interviews (closed questions), questionnaires and observation (checklists, etc.)[22].

Descriptive research is utilized to assess the proposed solution. The consequences of a descriptive analysis are exhibited as form of frequency tables and bar chart [20]. Oppenheim [20] talks about that the descriptive research does not lead to demonstrate a relationship between variables yet its goal is to present what proportion of a sample has a definite viewpoint. The survey is the most suitable research technique to approve the extent of this study since it is broadly connected with research on software engineering and it can collect data from an expanding number of respondents when contrasted with other research strategies. Moreover, Likert scale is utilized to catch the reactions as follow. Likert Scale Key: $1=$ Strongly Agree (SA), $2=$ Agree (A), $3=$ Neutral (N), 4 = Disagree (D), 5 = Strongly Disagree (SD).

A survey is conducted online, according to the recommendation of Nardi [21] to maintain a strategic distance from a human mistake in data collection and analysis. The survey comprised of thirty close-ended questions. Questions 1-5 are identified for the integration of the agile in the organization's policy so as to make them clear to everybody that the organization will embrace this strategy as the fundamental foundation for accomplishing their long term goals. Questions 69 are the manner by which to adjust resources to the organization to accomplish vital goals. These goals can be accomplished by learning and actualizing a coordinated culture in the organization. This group of questions additionally tries to learn the revolution of resources and what number of resources will be adequate whenever that ought to be in this procedure. Execution of the procedure is talked about in detail where the most imperative part of this execution is the precisely finding the learning necessities. Questions 10-15 are intended to support the continuous learning and its requirements and these requirements are discovered from various sources. New and rising advancements have the principal impact on the learning requirements, in this way, these must be embraced into thought before concluding the vital learning necessities. Execution likewise has another vital movement that is an action of discovering learning wishes. To discover learning necessities in a coordinated way, one ought to profit by the broad way of organizations and simple open access among different partners in the agile process. Consequently, learning longings can be found out amid these broad gatherings. Questions 16-19 are about the choice of the member of the team and afterward sending them in the team or outside for learning for leadership. Questions 20-25 are about the importance of managing knowledge and to know the significance of the steps to manage the knowledge. Question 25-30 are identified with the computation of the impacts of our methodology, to see whether this procedure will profit organization to raise it as the market pioneer. Impacts which are portrayed in this arrangement of inquiries reaches from the individual upgrade to group auxiliary impact and after that general impact of this methodology. This without a doubt will prompt the learning for leadership. 


\subsection{Cumulative Analysis Of OBJective 1.}

The cumulative analysis of every objective will be performed by taking the total of responses divided by the number of questions for every objective so the cumulative responses of the objective 1, 'Agile as integral part of the organization's policy' are shown in Table 1. $14.8 \%$ agreed while $22 \%$ strongly agreed to the agile as an integral part of the organization's policy. $7.6 \%$ of the cumulative responses were neutral. $5 \%$ of the respondents disagreed and $0.6 \%$ of the software engineers strongly disagreed to objective 1.

Table 1. Cumulative analysis of objective 1.

\begin{tabular}{|l|l|l|l|l|l|}
\hline Q. No. & $\begin{array}{l}\text { Str. } \\
\text { Disagree }\end{array}$ & Disagree & Neutral & Agree & $\begin{array}{l}\text { Str. } \\
\text { Agree }\end{array}$ \\
\hline 1 & 0 & 5 & 10 & 12 & 23 \\
\hline 2 & 0 & 1 & 5 & 16 & 28 \\
\hline 3 & 0 & 8 & 9 & 13 & 20 \\
\hline 4 & 2 & 5 & 5 & 15 & 23 \\
\hline 5 & 1 & 6 & 9 & 18 & 16 \\
\hline Total & 3 & 25 & 38 & 74 & 110 \\
\hline Average & 0.6 & 5 & 7.6 & 14.8 & 22 \\
\hline
\end{tabular}

\subsection{CuMulative ANAlysis OF OBJECTIVE 2.}

Cumulative responses of the objective 2, 'Allocates appropriate time and resources as the demand of the organization and its teams and measure their effect on the learning', were shown in Table 2. According to Table 2, 29.5\% were strongly agreed and $12.57 \%$ were agreed to it. $4.5 \%$ of the respondents disagreed while $0.75 \%$ of the participants were strongly disagreed to Goal 2 . Responses which remained neutral were $5 \%$.

Table 2. Cumulative analysis of objective 2

\begin{tabular}{|l|l|l|l|l|l|}
\hline Q. No. & $\begin{array}{l}\text { Str. } \\
\text { Disagree }\end{array}$ & Disagree & Neutral & Agree & $\begin{array}{l}\text { Str. } \\
\text { Agree }\end{array}$ \\
\hline 6 & 1 & 7 & 7 & 11 & 24 \\
\hline 7 & 1 & 3 & 4 & 12 & 30 \\
\hline 8 & 0 & 5 & 5 & 10 & 30 \\
\hline 9 & 1 & 3 & 4 & 18 & 34 \\
\hline Total & 3 & 18 & 20 & 51 & 118 \\
\hline Average & 0.6 & 4.5 & 5 & 12.75 & 29.5 \\
\hline
\end{tabular}

\subsection{Cumulative Analysis Of ObJective 3.}

Cumulative responses of the objective 3 were shown in Table 3. Table 3 showed that $16.8 \%$ of the responses agreed to objective 3 in which $19.8 \%$ of the software engineers strongly agreed and $16.8 \%$ of the professionals agreed to it. $8.5 \%$ of the responses remained neutral for Goal 3. $4.17 \%$ of the participants disagreed while $0.67 \%$ of the responses strongly disagreed to objective 3 . 
International Journal of Software Engineering \& Applications (IJSEA), Vol.8, No.6, November 2017

Table 3. Cumulative analysis of objective 3

\begin{tabular}{|l|l|l|l|l|l|}
\hline Q. No. & $\begin{array}{l}\text { Str. } \\
\text { Disagree }\end{array}$ & Disagree & Neutral & Agree & $\begin{array}{l}\text { Str. } \\
\text { Agree }\end{array}$ \\
\hline 10 & 1 & 3 & 10 & 17 & 19 \\
\hline 11 & 0 & 3 & 6 & 23 & 18 \\
\hline 12 & 1 & 7 & 5 & 19 & 18 \\
\hline 13 & 0 & 8 & 7 & 13 & 22 \\
\hline 14 & 2 & 2 & 9 & 13 & 24 \\
\hline 15 & 0 & 2 & 14 & 16 & 18 \\
\hline Total & 4 & 25 & 51 & 101 & 119 \\
\hline Average & 0.67 & 4.17 & 8.5 & 16.8 & 19.8 \\
\hline
\end{tabular}

\subsection{Cumulative Analysis Of OBJective 4.}

Cumulative responses of the objective 4, 'Measuring the effect of learning from leadership to the other teams in the organization', were shown in Table $4.21 .25 \%$ of the respondents strongly agreed while $12.5 \%$ of the professionals agreed to it. $9 \%$ of the responses remained neutral. $6.25 \%$ of the respondents disagreed while $1.25 \%$ of the software engineers strongly disagreed with the objective 4 .

Table 4. Cumulative analysis of objective 4

\begin{tabular}{|l|l|l|l|l|l|}
\hline Q. No. & $\begin{array}{l}\text { Str. } \\
\text { Disagree }\end{array}$ & Disagree & Neutral & Agree & $\begin{array}{l}\text { Str. } \\
\text { Agree }\end{array}$ \\
\hline 16 & 0 & 2 & 11 & 12 & 25 \\
\hline 17 & 1 & 9 & 8 & 18 & 14 \\
\hline 18 & 3 & 11 & 8 & 11 & 17 \\
\hline 19 & 1 & 3 & 9 & 9 & 29 \\
\hline Total & 5 & 25 & 36 & 50 & 85 \\
\hline Average & 1.25 & 6.25 & 9 & 12.5 & 21.25 \\
\hline
\end{tabular}

\subsection{Cumulative Analysis Of OBJective 5.}

Cumulative responses of the objective 5, 'Measuring the effect of the steps to manage the knowledge and its significant on Spread the learning', were shown in Table 5. Table 5 showed $21 \%$ of the respondents strongly agreed while $17.5 \%$ of the professionals agreed to it. $5.2 \%$ of the responses remained neutral. 5.5\% of the respondents disagreed while $0.83 \%$ of the software engineers strongly disagreed with the objective 5 . 
International Journal of Software Engineering \& Applications (IJSEA), Vol.8, No.6, November 2017

Table 5. Cumulative analysis of objective 5

\begin{tabular}{|l|l|l|l|l|l|}
\hline Q. No. & $\begin{array}{l}\text { Str. } \\
\text { disagree }\end{array}$ & Disagree & Neutral & Agree & $\begin{array}{l}\text { Str. } \\
\text { Agree }\end{array}$ \\
\hline 20 & 1 & 4 & 5 & 13 & 27 \\
\hline 21 & 2 & 6 & 6 & 19 & 17 \\
\hline 22 & 0 & 8 & 5 & 16 & 21 \\
\hline 23 & 2 & 1 & 3 & 25 & 19 \\
\hline 24 & 0 & 7 & 6 & 16 & 21 \\
\hline 25 & 0 & 7 & 6 & 16 & 21 \\
\hline Total & 5 & 33 & 31 & 105 & 126 \\
\hline Average & 0.83 & 5.5 & 5.2 & 17.5 & 21 \\
\hline
\end{tabular}

\subsection{Cumulative Analysis Of ObJective 6.}

Cumulative responses of the objective 6, 'Assessing the implementing of the proposed model to achieve the long term learning of whole teams in a medium and large organization. ', were shown in Table 6 . Table 6 showed that $17.2 \%$ of the respondents strongly agreed while $20.8 \%$ of the professionals agreed to it. $6 \%$ of the responses remained neutral. $4.6 \%$ of the respondents disagreed while $0.2 \%$ of the software engineers strongly disagreed with the objective 6 .

Table 6. Cumulative analysis of objective 6

\begin{tabular}{|l|l|l|l|l|l|}
\hline Q. No. & $\begin{array}{l}\text { Str. } \\
\text { Disagree }\end{array}$ & Disagree & Neutral & Agree & $\begin{array}{l}\text { Str. } \\
\text { Agree }\end{array}$ \\
\hline 26 & 0 & 3 & 2 & 21 & 24 \\
\hline 27 & 0 & 11 & 8 & 13 & 18 \\
\hline 28 & 1 & 1 & 9 & 16 & 23 \\
\hline 29 & 0 & 3 & 2 & 34 & 11 \\
\hline 30 & 0 & 5 & 9 & 20 & 10 \\
\hline Total & 1 & 23 & 30 & 104 & 86 \\
\hline Average & 0.2 & 4.6 & 6 & 20.8 & 17.2 \\
\hline
\end{tabular}

The final cumulative evaluation of objectives from 1 through 6 was shown in Table 7. So this work is supported with validation in which $43 \%$ people supported it while $10 \%$ disagreed to it as shown in "Figure. 6".

Table 7. Cumulative analysis of objectives 1-6

\begin{tabular}{|l|l|l|}
\hline Q.no & Choice & Percentage \\
\hline 1 & 639 & $43 \%$ \\
\hline 2 & 485 & $32 \%$ \\
\hline 3 & 206 & $14 \%$ \\
\hline 4 & 149 & $10 \%$ \\
\hline 5 & 21 & $1 \%$ \\
\hline TOTAL & 1500 & $100 \%$ \\
\hline
\end{tabular}


International Journal of Software Engineering \& Applications (IJSEA), Vol.8, No.6, November 2017

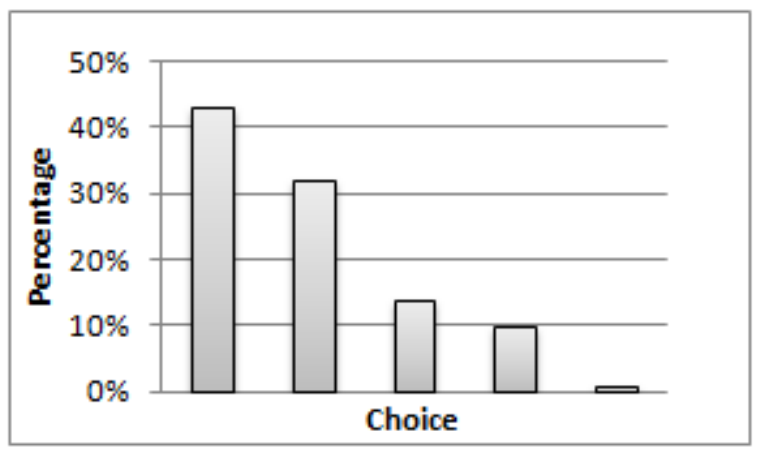

Figure 3. Graph showing the overall results of the validation of the proposed solution.

\subsection{COMPARATIVE STUdY}

This section will display comparison between the proposed adaptive, agile method with other proposed models that was studied in literature review and what the criteria that was investigated for team of every model.

In the Table 8, we observed the proposed model in this study take into consideration the quality of teamwork from coordination and communication and the team must have the strategic to be learned on long term also the consideration of knowledge management.

Table 8. Comparative study

\begin{tabular}{|c|c|c|c|}
\hline \multirow{2}{*}{$\begin{array}{l}\text { The proposed } \\
\text { models }\end{array}$} & \multicolumn{3}{|l|}{ Criteria } \\
\hline & Knowledge management & Teamwork quality & $\begin{array}{ll}\text { Long } \\
\text { learning }\end{array}$ \\
\hline [6] & & $\sqrt{ }$ & \\
\hline [7] & $\sqrt{ }$ & $\sqrt{ }$ & \\
\hline [13] & $\sqrt{ }$ & & \\
\hline [14] & $\sqrt{ }$ & & \\
\hline [24] & & $\sqrt{ }$ & $\sqrt{ }$ \\
\hline $\begin{array}{l}\text { The proposed } \\
\text { model }\end{array}$ & $\sqrt{ }$ & $\sqrt{ }$ & $\sqrt{ }$ \\
\hline
\end{tabular}

\section{Conclusion}

To scale suitable agile methodologies for a long-term basis, The adaptive framework is inserted in strategic management documents. Long-term implementation of agile, proper ownership, transparency and clarity will be provided by the document of strategic management. Then to accommodate rotational learning for leadership, the cross-functional agile team has altered with the introduction of a learner. Refinement of leadership qualities and continuous focus are necessary to have the capacity to go as a leader. The study emphasis on the technical aspects of leadership and other aspects, such as presentation skills, coordination skills, communication skills, satisfaction and motivation. The validation of the proposed framework is supported by $43 \%$ of the respondents. The proposed framework results indicate to scale agile methodologies 
International Journal of Software Engineering \& Applications (IJSEA), Vol.8, No.6, November 2017

are applicable to medium and large organizations. To scale agile methodologies and enhance the productivity of the organization the presented work will stimulate target companies to carry out the new adaptive framework.

\section{REFERENCES}

[1] L. M. R. Haraldsen, "An Investigation of Team Effectiveness in Agile Software Development," Institutt for datateknikk og informasjonsvitenskap, 2012.

[2] Whitworth, Elizabeth. "Agile experience: communication and collaboration in agile software development teams". ProQuest, 2006.

[3] J. A. Crowder and S. Friess, "Understanding the Agile Team," in Agile Project Management: Managing for Success, Cham: Springer International Publishing, 2015, pp. 27-41.

[4] K. Sutling, Z. Mansor, S. Widyarto, S. Letchmunan, and N. H. Arshad, "Agile project manager behavior: The taxonomy," in Software Engineering Conference (MySEC), 2014 8th Malaysian, 2014, pp. 234-239.

[5] E. van Kelle, J. Visser, A. Plaat, and P. van der Wijst, "An Empirical Study into Social Success Factors for Agile Software Development," 2015, pp. 77-80.

[6] I. Inayat and S. S. Salim, "A framework to study requirements-driven collaboration among agile teams: Findings from two case studies," Comput. Hum. Behav., vol. 51, pp. 1367-1379, Oct. 2015.

[7] Ibrahim A Elgendy, Mohamed El-kawkagy, and Arabi Keshk, "Improving the Performance of Mobile Applications Using Cloud Computing," in Informatics and Systems (INFOS), 2014 9th International Conference on, Cairo,2014, pp. 109-115.

[8] C. Melo, D. S. Cruzes, F. Kon, and R. Conradi, "Agile Team Perceptions of Productivity Factors," 2011, pp. 57-66.

[9] C. de O. Melo, D. S. Cruzes, F. Kon, and R. Conradi, "Interpretative case studies on agile team productivity and management," Inf. Softw. Technol., vol. 55, no. 2, pp. 412-427, Feb. 2013.

[10] R. Hoda, J. Noble, and S. Marshall, "Supporting self-organizing agile teams," in Agile Processes in Software Engineering and Extreme Programming, Springer, 2011, pp. 73-87.

[11] R. Hoda, J. Noble, and S. Marshall, "Self-Organizing Roles on Agile Software Development Teams," IEEE Trans. Softw. Eng., vol. 39, no. 3, pp. 422-444, Mar. 2013.

[12] C. Lassenius, T. Dingsøyr, and M. Paasivaara, Eds., Agile Processes, in Software Engineering, and Extreme Programming, vol. 212. Cham: Springer International Publishing, 2015.

[13] X. Yin, G. Zhu, and L. Feng, "Agile team learning model based on fast task mining," in New Horizons in Web-Based Learning-ICWL 2010 Workshops, 2011, pp. 328-335.

[14] R. K. Kavitha and M. I. Ahmed, "A knowledge management framework for agile software development teams," in Process Automation, Control and Computing (PACC), 2011 International Conference on, 2011, pp. 1-5.

[15] M. Mohamed, M. Stankosky, and A. Murray, "Applying knowledge management principles to enhance cross-functional team performance," J. Knowl. Manag., vol. 8, no. 3, pp. 127-142, Jun. 2004.

[16] D. Stankovic, V. Nikolic, M. Djordjevic, and D.-B. Cao, "A survey study of critical success factors in agile software projects in former Yugoslavia IT companies," J. Syst. Softw., vol. 86, no. 6, pp. 16631678, Jun. 2013.

[17] N. B. Moe, T. Dingsøyr, and T. Dybla a, "Overcoming barriers to self-management in software teams," IEEE Softw., vol. 26, no. 6, 2009.

[18] N. Salin and others, "Supporting proactivity in agile project teams through self organizing and shared leadership," 2017.

[19] J. McAvoy and T. Butler, "The role of project management in ineffective decision making within Agile software development projects,” Eur. J. Inf. Syst., vol. 18, no. 4, pp. 372-383, 2009.

[20] Faculty of Computing and Information Technology, King Abdulaziz University, Jeddah, Saudi Arabia, M. R. Jameel Qureshi, and M. Kashif, "Adaptive Framework to Manage Multiple Teams Using Agile Methodologies,” Int.J. Mod. Educ. Comput. Sci., vol. 9, no. 1, pp. 52-59, Jan. 2017.

[21] Nardi, P., 'Doing Survey Research: a Guide to Quantitative Methods' (Pearson Education, 2003). 
International Journal of Software Engineering \& Applications (IJSEA), Vol.8, No.6, November 2017

[22 http://research-methodology.net/research-methodology/research-design/conclusiveresearch/descriptive-research/

[23] M. Lamoreux, "Improving agile team learning by improving team reflections [agile software development]," in Agile Conference, 2005. Proceedings, 2005, pp. 139-144.

[24] M. R. J. Qureshi and M. Kashif, "Seamless long term learning in agile teams for sustainable leadership," in Emerging Technologies, 2009. ICET 2009. International Conference on, 2009, pp. 389-394.

\section{AUTHORS}

Dr. M. Rizwan Jameel Qureshi received his Ph.D. degree from National College of Business Administration \& Economics, Pakistan 2009. He is currently working as an Associate Professor in the Department of IT, King Abdulaziz University, Jeddah, Saudi Arabia. This author is the best researcher awardees from the Department of Information Technology, King Abdulaziz University in 2013 and the Department of Computer Science, COMSATS Institute of Information Technology, Lahore, Pakistan in 2008.

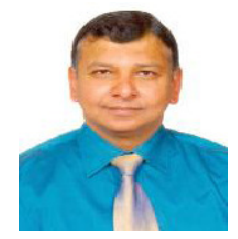

Zahra Abass completed his bachelor degree in information system from King Khaled University, Abha, Saudi Arabia. She is currently completing her master degree in the Department of IT, King Abdulaziz University, Jeddah, Saudi Arabia. 